\title{
Analysis of a Fractional Reaction-Diffusion HBV Model with Cure of Infected Cells
}

\author{
Moussa Bachraoui, ${ }^{1}$ Khalid Hattaf $\mathbb{D}^{1,2}$ and Noura Yousfi $\mathbb{D}^{1}$ \\ ${ }^{1}$ Laboratory of Analysis, Modeling and Simulation (LAMS), Faculty of Sciences Ben M'sik, Hassan II University of Casablanca, \\ P.O. Box 7955 Sidi Othman, Casablanca, Morocco \\ ${ }^{2}$ Centre Régional des Métiers de l'Education et de la Formation (CRMEF), Derb Ghalef, Casablanca 20340, Morocco
}

Correspondence should be addressed to Khalid Hattaf; k.hattaf@yahoo.fr

Received 22 May 2020; Revised 30 June 2020; Accepted 9 July 2020; Published 12 November 2020

Academic Editor: Fahd Jarad

Copyright ( 2020 Moussa Bachraoui et al. This is an open access article distributed under the Creative Commons Attribution License, which permits unrestricted use, distribution, and reproduction in any medium, provided the original work is properly cited.

\begin{abstract}
In this paper, we propose a fractional reaction-diffusion model in order to better understand the mechanisms and dynamics of hepatitis B virus (HBV) infection in human body. The infection transmission is modeled by Hattaf-Yousfi functional response, and the fractional derivative is in the sense of Caputo. The global stability of the model equilibria is analyzed by means of Lyapunov functionals. Finally, numerical simulations are presented to support our analytical results.
\end{abstract}

\section{Introduction}

In the recent years, fractional calculus has attracted the attention of many researchers. Hattaf [1] proposed a new fractional derivative with nonsingular kernel which generalizes many forms existing in the literature such as the Caputo-Fabrizio and Atangana-Baleanu fractional derivatives. Furthermore, there are some new methods used to solve numerically fractional models considered to explain deeper investigations of real-world problems [2-6].

On the other hand, hepatitis $B$ is a dangerous infectious disease caused by the hepatitis B virus (HBV). It affects the lives of 257 million people and responsible for the deaths of 56000 people every year according to World Health Organization (WHO) estimates [7]. Therefore, several mathematical models have been proposed and developed to describe the dynamics of HBV infection. For instance, Manna and Chakrabarty [8] proposed and analyzed the dynamics of HBV infection by taking into account the spacial mobility of both HBV DNA-containing capsids and viruses. Their work was an extension of that presented in [9]. Guo et al. [10] studied a nonlinear system of partial differential equations (PDEs) for HBV infection with three time delays, general incidence rate, and spatial diffusion only in the viruses. Hattaf and Yousfi [11] developed a mathematical HBV infection model with two modes of transmission, which allows the movement of HBV DNA-containing capsids and viruses, and three distributed delays. Since fractional-order models possess property of memory, Bachraou et al. [12] proposed a mathematical model governed by fractional differential equations (FDEs) to more explore the dynamic characteristics of the HBV infection. They have improved and generalized the ordinary differential equation (ODE) models $[9,13]$ and also the FDE models [14-16] by using Hattaf's incidence rate [17] that includes the common types such as the bilinear incidence rate, the saturated incidence rate, and the Beddington-DeAngelis functional response $[18,19]$.

In this study, we present an extension of our model presented in [12] by considering the mobility of capsids and viruses. So, we propose the following mathematical model formulated by fractional partial differential equations (FPDEs) to better describe the dynamics of HBV infection under the effects of diffusion and memory: 


$$
\left\{\begin{array}{l}
\partial_{t}^{\alpha} U=\sigma-\delta U(x, t)-F(U(x, t), V(x, t)) V(x, t)+\varepsilon I(x, t), \\
\partial_{t}^{\alpha} I=F(U(x, t), V(x, t)) V(x, t)-(\rho+\varepsilon) I(x, t), \\
\partial_{t}^{\alpha} C=d_{C} \Delta C+\kappa I(x, t)-(\rho+\eta) C(x, t), \\
\partial_{t}^{\alpha} V=d_{V} \Delta V+\eta C(x, t)-\nu V(x, t) .
\end{array}\right.
$$

The state variables $U(x, t), I(x, t), C(x, t)$, and $V(x, t)$ are, respectively, the concentrations of uninfected liver cells, infected liver cells, and HBV DNA-containing capsids and virions at location $x$ and time $t$. Uninfected liver cells are produced at constant rate $\sigma$, die at rate $\delta U$, and become infected by virus at rate $F(U, V) V$. The parameters $\varepsilon, \rho, \kappa, \eta$, and $\nu$ are, respectively, the cure rate of infected liver cells, the death rate of infected liver cells and capsids, the production rate of capsids from infected liver cells, the rate at which the capsids are converted to virions, and the viral clearance rate. The positive constants $d_{C}$ and $d_{V}$ are the diffusion coefficients of capsids and virus. $\Delta=\sum_{i=1}^{n} \partial^{2} / \partial x_{i}^{2}$ is the Laplacian operator. The incidence function of (1) is described by Hattaf-Yousfi functional response [17] of the form $F(U, V)=\left((b U) /\left(\alpha_{0}+\alpha_{1} U+\alpha_{2} V+\alpha_{3} U V\right)\right)$, where the nonnegative constants $\alpha_{i}, i=0,1,2,3$, measure the saturation, inhibitory, or psychological effects and the positive constant $b$ is the infection rate. This functional response covers several specific cases available in the literature such as the bilinear and saturation incidences, the Beddington-DeAngelis and Crowley-Martin functional responses, and the specific functional response introduced by Hattaf et al. [20]. Finally, $\partial_{t}^{\alpha}$ is the Caputo fractional derivative of order $\alpha \in(0,1]$. The choice of this type of fractional derivative is motivated by the fact that the fractional derivative of a constant is equal to zero, and $\alpha$ was chosen in the interval $(0,1]$ to have the same initial conditions as those of the PDE systems. Furthermore, a recent study in [21] has shown that the fractional-order model gives better predictions than that of the integer model about the plasma viral load of the patients.

Throughout this paper, we consider system (1) with initial conditions

$$
\begin{gathered}
U(x, 0)=U_{0}(x) \geq 0, \\
I(x, 0)=I_{0}(x) \geq 0, \\
V(x, 0)=V_{0}(x) \geq 0, \\
C(x, 0)=C_{0}(x) \geq 0,
\end{gathered}
$$

$$
\forall x \in \bar{\Omega},
$$

and zero-flux boundary conditions

$$
\frac{\partial C}{\partial \vec{n}}=\frac{\partial V}{\partial \vec{n}}=0, \quad \text { on } \partial \Omega \times(0,+\infty),
$$

where $\Omega$ is a bounded domain in $\mathbb{R}^{n}$ with smooth boundary $\partial \Omega$ and $\partial / \partial \vec{n}$ denotes the outward normal derivative on $\partial \Omega$. From the biological point of view, these boundary conditions indicate that the capsids and free viral particles do not move across the boundary $\partial \Omega$.
The rest of the paper is organized as follows. The following section is devoted to the calculations of the basic reproduction number and steady states of model (1). The global dynamics of the FPDE model is analyzed in Section 3. To support the analytical results, we present some numerical simulations in Section 4. Finally, we end the paper with biological and mathematical conclusions in Section 5.

\section{Equilibria of the FPDE Model}

It is easy to verify that the only infection-free steady state of the FPDE model $(1)$ is $P_{0}\left(U^{0}, 0,0,0\right)$, where $U^{0}=(\sigma / \delta)$. Then, the basic reproduction number of (1) is given by

$$
\mathscr{R}_{0}=\frac{\kappa \eta F\left(U^{0}, 0\right)}{\nu(\eta+\rho)(\varepsilon+\rho)} .
$$

The other steady states verify the following system:

$$
\begin{aligned}
\sigma-\delta U-F(U, V) V+\varepsilon I & =0, \\
F(U, V) V-(\varepsilon+\rho) I & =0, \\
\kappa I-(\eta+\rho) C & =0, \\
\eta C-\nu V & =0 .
\end{aligned}
$$

From $(5)-(8)$, we obtain $I=((\sigma-\delta U) / \rho), C=((\kappa(\sigma-$ $\delta U)) /(\rho(\eta+\rho))), V=((\eta \kappa(\sigma-\delta U)) /(\nu \rho(\eta+\rho)))$, and

$$
F\left(U, \frac{\eta \kappa(\sigma-\delta U)}{\nu \rho(\eta+\rho)}\right)=\frac{\nu(\eta+\rho)(\rho+\varepsilon)}{\kappa \eta} .
$$

$I=((\sigma-\delta U) / \rho) \geq 0$ implies that $U \leq(\sigma / \delta)$. So, we consider the function $G$ defined on interval $[0,(\sigma / \delta)]$ by

$$
G(U)=F\left(U, \frac{\eta \kappa(\sigma-\delta U)}{\nu \rho(\eta+\rho)}\right)-\frac{\nu(\eta+\rho)(\rho+\varepsilon)}{\kappa \eta} .
$$

We have $G(0)=-((\nu(\eta+\rho)(\rho+\varepsilon)) / \kappa \eta)<0, G(\sigma / \delta)=$ $((\nu(\eta+\rho)(\rho+\varepsilon)) / \kappa \eta)\left(\mathscr{R}_{0}-1\right)$, and

$$
G^{\prime}(U)=\frac{\partial F}{\partial U}-\frac{\kappa \eta \delta}{\nu \rho(\eta+\rho)} \frac{\partial F}{\partial V}>0 .
$$

If $\mathscr{R}_{0}>1$, we deduce that system (1) admits a unique infection equilibrium $P_{1}\left(U_{1}, I_{1}, C_{1}, V_{1}\right)$ with $U_{1} \in(0$, $(\sigma / \delta)), \quad I_{1}=\left(\left(\sigma-\delta U_{1}\right) / \rho\right), \quad C_{1}=\left(\kappa\left(\sigma-\delta U_{1}\right)\right) /(\rho(\eta+\rho))$, and $V_{1}=\left(\eta \kappa\left(\sigma-\delta U_{1}\right)\right) /(\nu \rho(\eta+\rho))$.

We summarize the above discussions in the following result.

\section{Theorem 1}

(i) When $\mathscr{R}_{0} \leq 1$, the FPDE model (1) has one infectionfree steady state $P_{0}\left(U^{0}, 0,0,0\right)$, where $U^{0}=(\sigma / \delta)$

(ii) When $\mathscr{R}_{0}>1$, the FPDE model (1) has uniquely one chronic infection steady state $P_{1}\left(U_{1}, I_{1}, C_{1}, V_{1}\right)$, where $U_{1} \in(0,(\sigma / \delta)), I_{1}=\left(\left(\sigma-\delta U_{1}\right) / \rho\right), C_{1}=(\kappa$ $\left.\left(\sigma-\delta U_{1}\right)\right) /(\rho(\eta+\rho))$, and $V_{1}=\left(\eta \kappa\left(\sigma-\delta U_{1}\right)\right) /$ $(\nu \rho(\eta+\rho))$ 


\section{Global Dynamics}

This section analyzes the global dynamics of the FPDE model (1).

$$
L_{0}(t)=\int_{\Omega}\left[\frac{\alpha_{0}}{\alpha_{0}+\alpha_{1} U^{0}} U^{0} \Phi\left(\frac{U}{U^{0}}\right)+\frac{\alpha_{0} \varepsilon\left(U(x, t)-U^{0}+I(x, t)\right)^{2}}{2(\rho+\delta)\left(\alpha_{0}+\alpha_{1} U^{0}\right) U^{0}}+I(x, t)+\frac{\varepsilon+\rho}{\kappa} C(x, t)+\frac{(\varepsilon+\rho)(\eta+\rho)}{\kappa \eta} V(x, t)\right] \mathrm{d} x
$$

where $\Phi(x)=x-1-\ln (x)$ for $x>0$. According to [22], we obtain

$$
D^{\alpha} L_{0}(t) \leq \int_{\Omega}\left[\frac{\alpha_{0}}{\alpha_{0}+\alpha_{1} U^{0}}\left(1-\frac{U^{0}}{U}\right) \partial_{t}^{\alpha} U+\partial_{t}^{\alpha} I+\frac{\alpha_{0} \varepsilon\left(U-U^{0}+I\right)\left(\partial_{t}^{\alpha} U+\partial_{t}^{\alpha} I\right)}{(\rho+\delta)\left(\alpha_{0}+\alpha_{1} U^{0}\right) U^{0}} \frac{\varepsilon+\rho}{\kappa} \partial_{t}^{\alpha} C+\frac{(\varepsilon+\rho)(\eta+\rho)}{\kappa \eta} \partial_{t}^{\alpha} V\right] \mathrm{d} x
$$

By $\sigma=\delta U^{0}$, we have

$$
\begin{aligned}
D^{\alpha} L_{0}(t) \leq & \int_{\Omega}\left[-\left(\frac{1}{U}+\frac{\varepsilon}{(\rho+\delta) U^{0}}\right) \frac{\alpha_{0} \delta\left(U-U^{0}\right)^{2}}{\alpha_{0}+\alpha_{1} U^{0}}-\frac{\alpha_{0} \varepsilon \rho I^{2}}{(\rho+\delta)\left(\alpha_{0}+\alpha_{1} U^{0}\right) U^{0}}\right. \\
& \left.-\frac{\alpha_{0} \varepsilon I\left(U-U^{0}\right)^{2}}{\left(\alpha_{0}+\alpha_{1} U^{0}\right) U^{0} U}+\frac{\nu(\varepsilon+\rho)(\eta+\rho)}{\kappa \eta}\left(\mathscr{R}_{0} \frac{F(U, V)}{F\left(U^{0}, 0\right)}-1\right) V\right] \mathrm{d} x, \\
\leq & -\int_{\Omega}\left[\left(\frac{1}{U}+\frac{\varepsilon}{(\rho+\delta) U^{0}}\right) \frac{\alpha_{0} \delta\left(U-U^{0}\right)^{2}}{\alpha_{0}+\alpha_{1} U^{0}}+\frac{\alpha_{0} \varepsilon \rho I^{2}}{(\rho+\delta)\left(\alpha_{0}+\alpha_{1} U^{0}\right) U^{0}}+\frac{\alpha_{0} \varepsilon I\left(U-U^{0}\right)^{2}}{\left(\alpha_{0}+\alpha_{1} U^{0}\right) U^{0} U}\right. \\
& \left.-\frac{\nu(\varepsilon+\rho)(\eta+\rho)}{\kappa \eta}\left(\mathscr{R}_{0}-1\right) V\right] \mathrm{d} x .
\end{aligned}
$$

Then, $D^{\alpha} L_{0}(t) \leq 0$ when $\mathscr{R}_{0} \leq 1$. In addition, $\left\{P_{0}\right\}$ is the largest invariant set in $\left\{(U, I, C, V) \mid D^{\alpha} L_{0}(t)=0\right\}$. By

$$
\mathscr{R}_{0} \leq 1+\frac{\left[\delta \rho \nu(\eta+\rho)+\alpha_{2} \delta \sigma \kappa \eta\right](\varepsilon+\rho)+\alpha_{3} \varepsilon \eta \kappa \sigma^{2}}{\varepsilon v \rho(\varepsilon+\rho)\left(\alpha_{0} \delta+\alpha_{1} \sigma\right)} .
$$
LaSalle's invariance principle [23], $P_{0}$ is globally asymptotically stable if $\mathscr{R}_{0} \leq 1$.

Theorem 3. The chronic infection steady state $P_{1}$ is globally asymptotically stable when $\mathscr{R}_{0}>1$ and
Proof. Let

$$
\begin{aligned}
L_{1}(t)= & \int_{\Omega}\left[\frac{\alpha_{0}+\alpha_{2} V_{1}}{\alpha_{0}+\alpha_{1} U_{1}+\alpha_{2} V_{1}+\alpha_{3} U_{1} V_{1}} U_{1} \Phi\left(\frac{U}{U_{1}}\right)+\frac{\varepsilon\left(\alpha_{0}+\alpha_{2} V_{1}\right)\left(U(x, t)-U_{1}+I(x, t)-I_{1}\right)^{2}}{2(\rho+\delta)\left(\alpha_{0}+\alpha_{1} U_{1}+\alpha_{2} V_{1}+\alpha_{3} U_{1} V_{1}\right) U_{1}}\right. \\
& \left.+I_{1} \Phi\left(\frac{I}{I_{1}}\right)+\frac{\varepsilon+\rho}{\kappa} C_{1} \Phi\left(\frac{C}{C_{1}}\right)+\frac{(\varepsilon+\rho)(\eta+\rho)}{\kappa \eta} V_{1} \Phi\left(\frac{V}{V_{1}}\right)\right] \mathrm{d} x .
\end{aligned}
$$


Then,

$$
\begin{aligned}
D^{\alpha} L_{1}(t) \leq & \int_{\Omega}\left[\frac{\alpha_{0}+\alpha_{2} V_{1}}{\alpha_{0}+\alpha_{1} U_{1}+\alpha_{2} V_{1}+\alpha_{3} U_{1} V_{1}}\left(1-\frac{U_{1}}{U}\right) \partial_{t}^{\alpha} U\right. \\
& +\frac{\varepsilon\left(\alpha_{0}+\alpha_{2} V_{1}\right)\left(U-U_{1}+I-I_{1}\right)\left(\partial_{t}^{\alpha} U+\partial_{t}^{\alpha} I\right)}{(\rho+\delta)\left(\alpha_{0}+\alpha_{1} U_{1}+\alpha_{2} V_{1}+\alpha_{3} U_{1} V_{1}\right) U_{1}}+\left(1-\frac{I_{1}}{I}\right) \partial_{t}^{\alpha} I \\
& \left.+\frac{\varepsilon+\rho}{\kappa}\left(1-\frac{C_{1}}{C}\right) \partial_{t}^{\alpha} C+\frac{(\varepsilon+\rho)(\eta+\rho)}{\kappa \eta}\left(1-\frac{V_{1}}{V}\right) \partial_{t}^{\alpha} V\right] \mathrm{d} x
\end{aligned}
$$

Since $\sigma=\delta U_{1}+\rho I_{1}, \quad F\left(U_{1}, V_{1}\right) V_{1}=(\varepsilon+\rho) I_{1}, \quad \kappa I_{1}=(\eta+$ $\rho) C_{1}$, and $1-\left(\left(F\left(U_{1}, V_{1}\right)\right) /\left(F\left(U, V_{1}\right)\right)\right)=\left(\left(\alpha_{0}+\alpha_{2} V_{1}\right) /\right.$ $\left.\left(\alpha_{0}+\alpha_{1} U_{1}+\alpha_{2} V_{1}+\alpha_{3} U_{1} V_{1}\right)\right)\left(1-\left(U_{1} / U\right)\right)$, we have

$$
\begin{aligned}
& D^{\alpha} L_{1}(t) \leq \int_{\Omega}\left[\frac{-\delta\left(\alpha_{0}+\alpha_{2} V_{1}\right)\left(U-U_{1}\right)^{2}}{\left(\alpha_{0}+\alpha_{1} U_{1}+\alpha_{2} V_{1}+\alpha_{3} U_{1} V_{1}\right) U}+\frac{\varepsilon\left(\alpha_{0}+\alpha_{2} V_{1}\right)\left(U-U_{1}\right)\left(I-I_{1}\right)}{\left(\alpha_{0}+\alpha_{1} U_{1}+\alpha_{2} V_{1}+\alpha_{3} U_{1} V_{1}\right) U}\right. \\
&+F\left(U, V_{1}\right) V_{1}\left(1-\frac{F\left(U_{1}, V_{1}\right)}{F\left(U, V_{1}\right)}+\frac{F(U, V) V_{1}}{F\left(U, V_{1}\right) V_{1}}\right)+F\left(U_{1}, V_{1}\right) V_{1}\left(1-\frac{I C_{1}}{I_{1} C}\right) \\
&+F\left(U_{1}, V_{1}\right) V_{1}\left(1-\frac{F(U, V) V I_{1}}{F\left(U_{1}, V_{1}\right) I V_{1}}\right)+F\left(U_{1}, V_{1}\right) V_{1}\left(1-\frac{V}{V_{1}}-\frac{C V_{1}}{C_{1} V}\right) \\
& \\
&\left.-\frac{\varepsilon\left(\alpha_{0}+\alpha_{2} V_{1}\right)\left[\delta\left(U-U_{1}\right)^{2}+\rho\left(I-I_{1}\right)^{2}+(\delta+\rho)\left(U-U_{1}\right)\left(I-I_{1}\right)\right]}{\left(\alpha_{0}+\alpha_{1} U_{1}+\alpha_{2} V_{1}+\alpha_{3} U_{1} V_{1}\right)(\delta+\rho) U_{1}}\right] \mathrm{d} x \\
&-\frac{\varepsilon+\rho}{\kappa} \mathrm{d}_{C} C_{1} \int_{\Omega} \frac{\|\nabla C\|^{2}}{C^{2}} \mathrm{~d} x-\frac{(\varepsilon+\rho)(\eta+\rho)}{\kappa \eta} \mathrm{d}_{V} V_{1} \int_{\Omega} \frac{\|\nabla V\|^{2}}{V^{2}} \mathrm{~d} x .
\end{aligned}
$$

Hence,

$$
\begin{aligned}
D^{\alpha} L_{1}(t) \leq & -\int_{\Omega}\left[\frac{\left(\alpha_{0}+\alpha_{2} V_{1}\right)\left(U-U_{1}\right)^{2}}{\left(\alpha_{0}+\alpha_{1} U_{1}+\alpha_{2} V_{1}+\alpha_{3} U_{1} V_{1}\right) U U_{1}}\left(\left(\delta U_{1}-\varepsilon I_{1}\right)+\frac{\delta \varepsilon U}{\rho+\delta}+\varepsilon I\right)\right. \\
& +\frac{\varepsilon \rho\left(\alpha_{0}+\alpha_{2} V_{1}\right)\left(I-I_{1}\right)^{2}}{\left(\alpha_{0}+\alpha_{1} U_{1}+\alpha_{2} V_{1}+\alpha_{3} U_{1} V_{1}\right)(\rho+\delta) U_{1}} \\
& -F\left(U_{1}, V_{1}\right) V_{1}\left(5-\frac{F\left(U_{1}, V_{1}\right)}{F\left(U, V_{1}\right)}-\frac{C_{1} I}{C I_{1}}-\frac{F(U, V)}{F\left(U_{1}, V_{1}\right)} \frac{V I_{1}}{V_{1} I}-\frac{C V_{1}}{C_{1} V}-\frac{F\left(U, V_{1}\right)}{F(U, V)}\right) \\
& \left.+\frac{F\left(U_{1}, V_{1}\right) V_{1}\left(\alpha_{0}+\alpha_{1} U\right)\left(\alpha_{2}+\alpha_{3} U\right)\left(V-V_{1}\right)^{2}}{\left(\alpha_{0}+\alpha_{1} U+\alpha_{2} V_{1}+\alpha_{3} U V_{1}\right)\left(\alpha_{0}+\alpha_{1} U+\alpha_{2} V+\alpha_{3} U V\right) V_{1}}\right] \mathrm{d} x \\
& -\frac{\varepsilon+\rho}{\kappa} \mathrm{d}_{C} C_{1} \int_{\Omega} \frac{\|\nabla C\|^{2}}{C^{2}} \mathrm{~d} x-\frac{(\varepsilon+\rho)(\eta+\rho)}{\kappa \eta} \mathrm{d}_{V} V_{1} \int_{\Omega} \frac{\|\nabla V\|^{2}}{V^{2}} \mathrm{~d} x .
\end{aligned}
$$



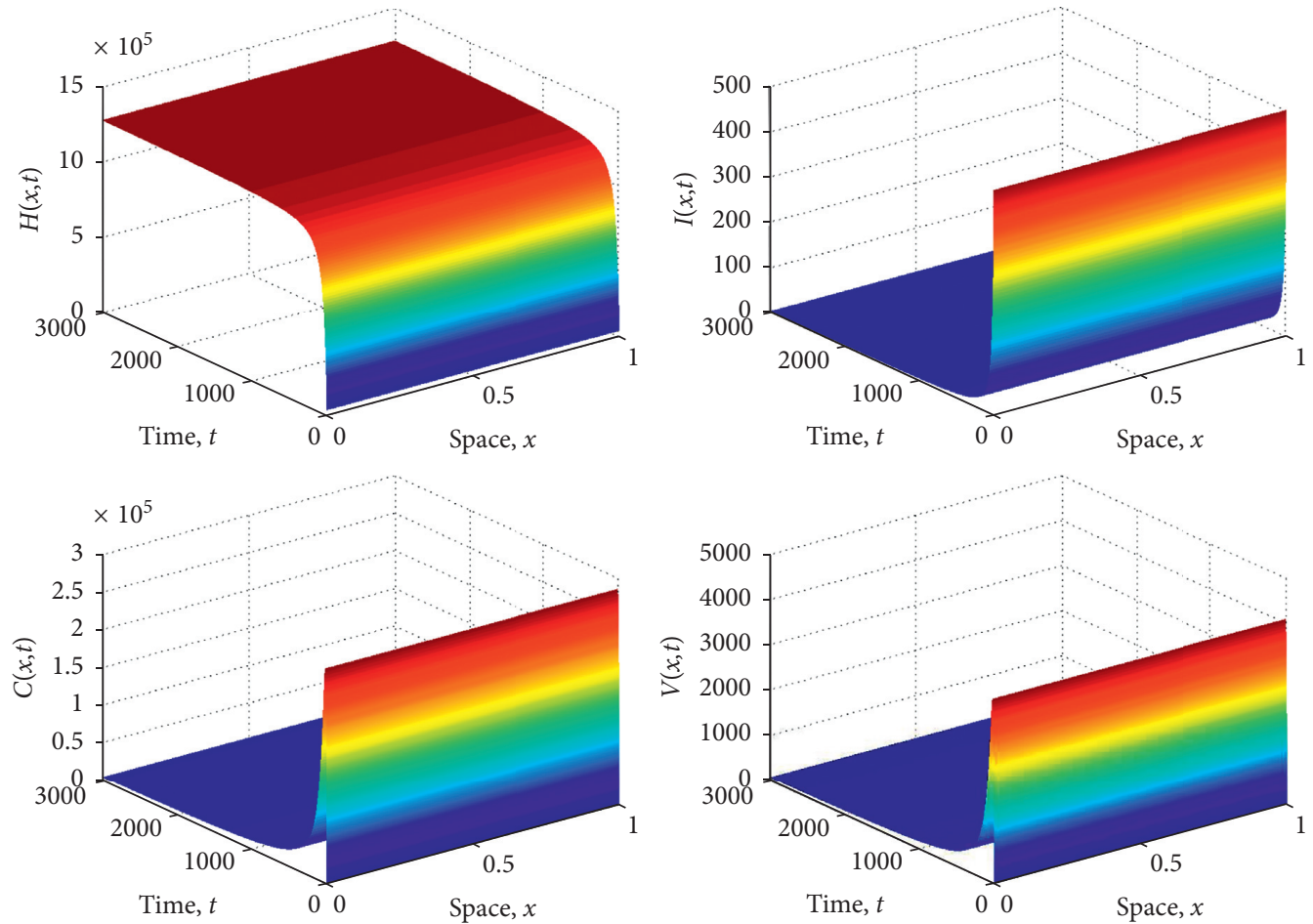

FIgURE 1: Stability of the infection-free steady state $P_{0}$.
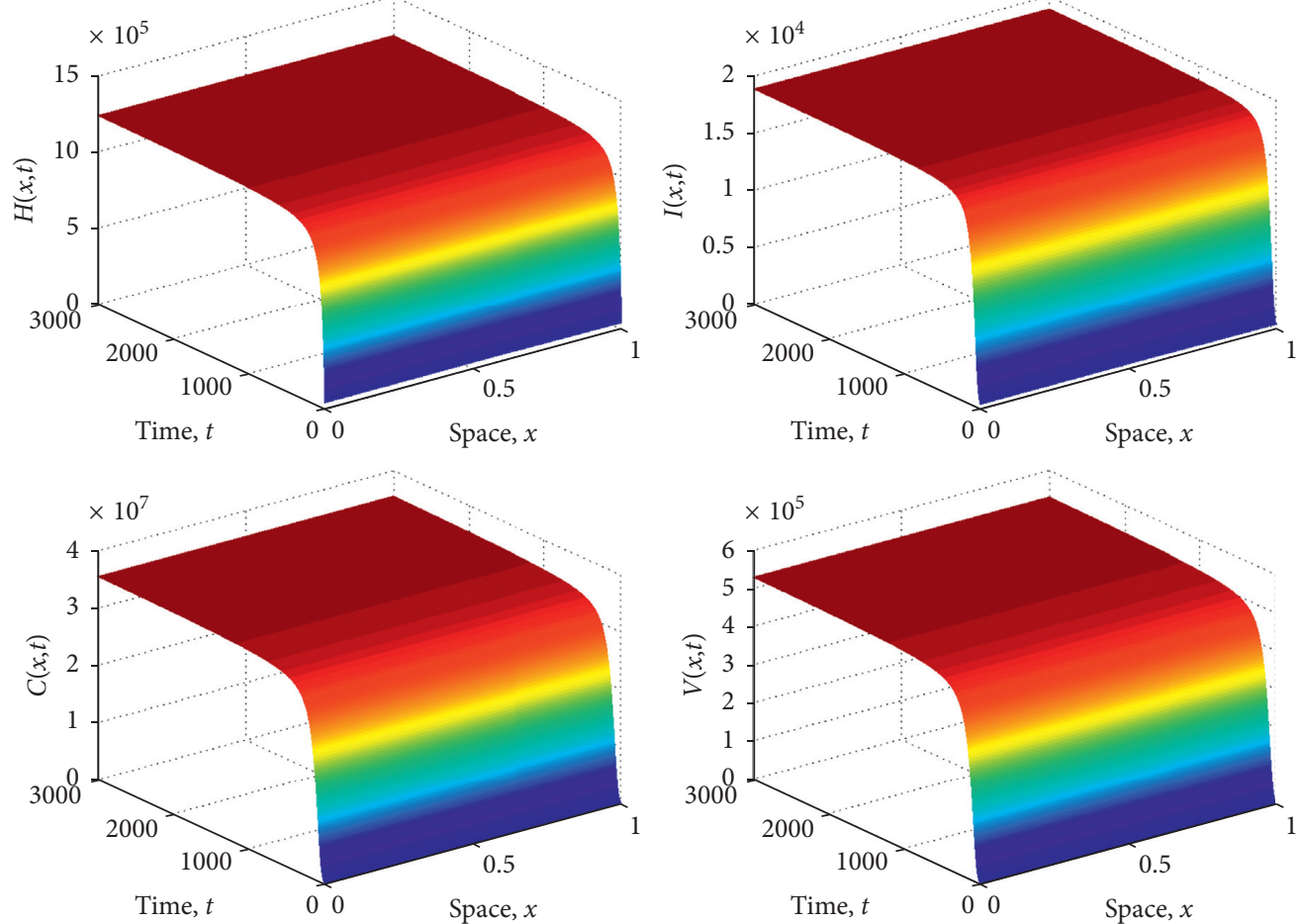

Figure 2: Stability of the chronic infection steady state $P_{1}$.

Since $\quad 5-\left(\left(F\left(U_{1}, V_{1}\right)\right) /\left(F\left(U, V_{1}\right)\right)\right)-\left(C_{1} I / C I_{1}\right)-((F$ $\left.(U, V)) /\left(F\left(U_{1}, V_{1}\right)\right)\right)\left(V I_{1} / V_{1} I\right)-\left(C V_{1} / C_{1} V\right)-\left(\left(F\left(U, V_{1}\right)\right) /\right.$ $(F(U, V))) \leq 0$, we have $D^{\alpha} L_{1}(t) \leq 0$ if $\mathscr{R}_{0}>1$ and $\varepsilon I_{1} \leq \delta U_{1}$. The last condition is equivalent to

$$
\mathscr{R}_{0} \leq 1+\frac{\left[\delta \rho \nu(\eta+\rho)+\alpha_{2} \delta \sigma \kappa \eta\right](\varepsilon+\rho)+\alpha_{3} \varepsilon \eta \kappa \sigma^{2}}{\varepsilon v \rho(\varepsilon+\rho)\left(\alpha_{0} \delta+\alpha_{1} \sigma\right)} .
$$



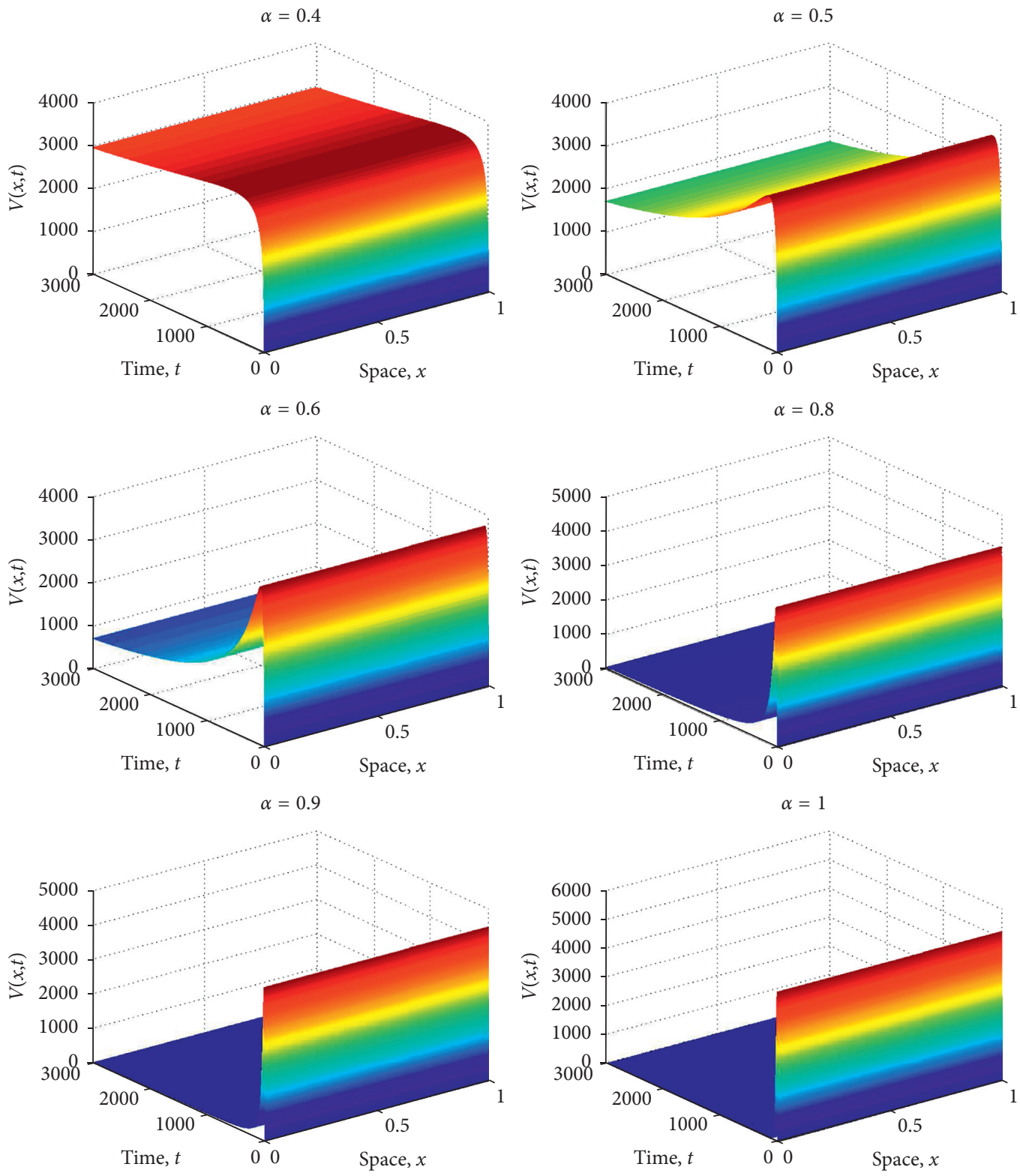

FIgUre 3: The state variable $V(x, t)$ with different values of $\alpha$.

Also, $\left\{P_{1}\right\}$ is the largest invariant set in $\{(U, I, C$, $\left.V) \mid D^{\alpha} L_{1}(t)=0\right\}$. According to LaSalle's invariance principle, we deduce that $P_{1}$ is globally asymptotically stable. This completes the proof.

\section{Numerical Simulations}

In this section, we present some numerical illustrations to support the obtained analytical results.

Let $\Delta t$ be the time step size, $\Omega=\left[x_{\min }, x_{\max }\right]$, and $\Delta x=$ $\left(x_{\max }-x_{\min }\right) / N$ be the space step size, where $N$ is a positive integer. The grid points for the space are $x_{i}=x_{\min }+i \Delta x$ for $i \in\{0, \ldots, N\}$ and for time are $t_{m}=m \Delta t$ for $m \in$ IN. From the Grünwald-Letnikov method [24], the Caputo fractional derivative is approximated as follows:

$$
{ }^{C} \partial_{t}^{\alpha} l\left(x_{i}, t_{m}\right) \approx \frac{1}{\Delta t^{\alpha}} \sum_{j=0}^{m} \beta_{j}^{\alpha} l\left(x_{i}, t_{m-j}\right)-\tilde{l}_{m},
$$

where $\tilde{l}_{m}=\left(\left(l\left(x_{i}, 0\right) t_{m}^{-\alpha}\right) /\left(\Gamma\left(17_{\alpha}^{\alpha}\right)\right)\right)$ and $\beta_{j}^{\alpha}$ are the fractional binomial coefficients $\left(\begin{array}{c}\alpha \\ j\end{array}\right)$ with the recursion

$$
\begin{aligned}
& \beta_{j}^{\alpha}=\left(1-\frac{1+\alpha}{j}\right) \beta_{j-1}^{\alpha}, \\
& \beta_{0}^{\alpha}=1 .
\end{aligned}
$$

Let $\left(U_{i}^{m}, I_{i}^{m}, C_{i}^{m}, V_{i}^{m}\right)$ be the approximations of the solution $(U, I, C, V)$ of $(1)$ at the discretized point $\left(x_{i}, t_{m}\right)$. Then, by applying (21), we obtain 


$$
\begin{aligned}
& \frac{1}{\Delta t^{\alpha}}\left(U_{i}^{m+1}+\sum_{j=1}^{m+1} \beta_{j i}^{\alpha} U^{m+1-j}\right)-\widetilde{U}_{i}^{m+1}=\sigma-\delta U_{i}^{m}-F\left(U_{i}^{m}, V_{i}^{m}\right) V_{i}^{m}+\varepsilon I_{i}^{m}, \\
& \frac{1}{\Delta t^{\alpha}}\left(I_{i}^{m+1}+\sum_{j=1}^{m+1} \beta_{j}^{\alpha} I_{i}^{m+1-j}\right)-\widetilde{I}_{i}^{m+1}=F\left(U_{i}^{m}, V_{i}^{m}\right) V_{i}^{m}-(\varepsilon+\rho) I_{i}^{m}, \\
& \frac{1}{\Delta t^{\alpha}}\left(C_{i}^{m+1}+\sum_{j=1}^{m+1} \beta_{j}^{\alpha} C_{i}^{m+1-j}\right)-\widetilde{C}_{i}^{m+1}=d_{C} \frac{C_{i+1}^{m}-2 C_{i}^{m}+C_{i-1}^{m}}{\Delta x^{2}} \kappa I_{i}^{m}-(\eta+\rho) C_{i}^{m}, \\
& \frac{1}{\Delta t^{\alpha}}\left(V_{i}^{m+1}+\sum_{j=1}^{m+1} \beta_{j}^{\alpha} V_{i}^{m+1-j}\right)-\widetilde{V}_{i}^{m+1}=d_{V} \frac{V_{i+1}^{m}-2 V_{i}^{m}+V_{i-1}^{m}}{\Delta x^{2}}+\eta C_{i}^{m}-\nu V_{i}^{m} .
\end{aligned}
$$

Hence,

$$
\begin{aligned}
& U_{i}^{m+1}=-\sum_{j=1}^{m+1} \beta_{j}^{\alpha} U_{i}^{m+1-j}+\Delta t^{\alpha}\left[\widetilde{U}_{i}^{m+1}+\sigma-\delta H_{i}^{m}-F\left(U_{i}^{m}, V_{i}^{m}\right) V_{i}^{m}+\varepsilon I_{i}^{m}\right], \\
& I_{i}^{m+1}=-\sum_{j=1}^{m+1} \beta_{j}^{\alpha} I_{i}^{m+1-j}+\Delta t^{\alpha}\left[\widetilde{I}_{i}^{m+1}+F\left(U_{i}^{m}, V_{i}^{m}\right) V_{i}^{m}-(\varepsilon+\rho) I_{i}^{m}\right], \\
& C_{i}^{m+1}=-\sum_{j=1}^{m+1} \beta_{j}^{\alpha} C_{i}^{m+1-j}+\Delta t^{\alpha}\left[\widetilde{C}_{i}^{m+1}+d_{C} \frac{C_{i+1}^{m}-2 C_{i}^{m}+C_{i-1}^{m}}{\Delta x^{2}}+\kappa I_{i}^{m}-(\eta+\rho) C_{i}^{m}\right], \\
& V_{i}^{m+1}=-\sum_{j=1}^{m+1} \beta_{j}^{\alpha} V_{i}^{m+1-j}+\Delta t^{\alpha}\left[\widetilde{V}_{i}^{m+1}+d_{V} \frac{V_{i+1}^{m}-2 V_{i}^{m}+V_{i-1}^{m}}{\Delta x^{2}} \eta C_{i}^{m}-\nu V_{i}^{m}\right] .
\end{aligned}
$$

For numerical simulations, we choose $\Omega=[0,1]$, $d_{C}=0.1, d_{V}=0.1, \quad \sigma=50400, \quad b=3.6 \times 10^{-6}, \delta=0.039$, $\rho=0.0693, \kappa=150, \eta=0.01, \varepsilon=0.01, \alpha=0.8, \alpha_{0}=0.1$, $\alpha_{1}=0.1, \alpha_{2}=0.01$, and $\alpha_{3}=0.000001$. By simple calculation, we find $\mathscr{R}_{0}=0.0128<1$. According to Theorem 2 , the infection-free steady state $P_{0}\left(1.2923 \times 10^{6}, 0,0,0\right)$ is globally asymptotically stable which means that the virus will disappear and the patient will be completely cured. Figure 1 confirms this result.

To numerically illustrate the global stability of the second steady state of model (1), we take $b=0.0018$ without changing the values of the other parameters. In this case, $\mathscr{R}_{0}=6.4083>1$. From Theorem 1, FPDE model (1) has the unique chronic infection steady state $P_{1}\left(1.244 \times 10^{6}\right.$, $\left.1.891 \times 10^{4}, 3.562 \times 10^{7}, 5.314 \times 10^{5}\right)$. In addition, we have

$$
1+\frac{\left[\delta \rho \nu(\eta+\rho)+\alpha_{2} \delta \sigma \kappa \eta\right](\varepsilon+\rho)+\alpha_{3} \varepsilon \eta \kappa \sigma^{2}}{\varepsilon \nu \rho(\varepsilon+\rho)\left(\alpha_{0} \delta+\alpha_{1} \sigma\right)}=218.9236
$$

which implies that (15) holds. By Theorem 3, $P_{1}$ is globally asymptotically stable. Figure 2 validates this result.

\section{Conclusions}

In this article, we have presented a fractional reaction-diffusion HBV model that takes into account the HBV DNAcontaining capsids and the cure of infected liver cells. The spatial diffusion is considered in capsids and virions, and the incidence of infection is described by Hattaf-Yousfi functional response that includes various forms existing in the literature. We have shown that the global dynamics of the FPDE model is fully determined by a threshold parameter called the basic reproduction number and labeled by $\mathscr{R}_{0}$. More concretely, the infection-free steady state $P_{0}$ is globally asymptotically stable if $\mathscr{R}_{0} \leq 1$, which biologically means that the HBV is cleared. However, the chronic infection steady state $P_{1}$ is globally asymptotically stable when $\mathscr{R}_{0}>1$ and condition (15) holds. In this case, the HBV persists in the liver and the infection becomes chronic.

According to the above analytic results and the numerical simulations, we deduce that the diffusion and the order of fractional derivative in sense of Caputo have no effects on the stability of both steady states, but they can affect the time for arriving to these steady states. For 
example, the trajectories quickly converge towards the equilibria of the model for higher values of the fractional derivative order (see, Figure 3). On the other hand, the models and results presented in $[8,9,12-16]$ are improved and extended.

\section{Data Availability}

The data used to support the findings of this study are available from the corresponding author upon request.

\section{Conflicts of Interest}

The authors declare that they have no conflicts of interest.

\section{References}

[1] K. Hattaf, "A new generalized definition of fractional derivative with non-singular kernel," Computation, vol. 8, no. 2, p. $49,2020$.

[2] K. M. Owolabi and Z. Hammouch, "Spatiotemporal patterns in the Belousov-Zhabotinskii reaction systems with Atangana-Baleanu fractional order derivative," Physica A: Statistical Mechanics and Its Applications, vol. 523, pp. 1072-1090, 2019.

[3] A. Yokuş and S. Gülbahar, "Numerical solutions with linearization techniques of the fractional harry dym equation," Applied Mathematics and Nonlinear Sciences, vol. 4, no. 1, pp. 35-42, 2019.

[4] W. Gao, P. Veeresha, D. G. Prakasha, H. M. Baskonus, and G. Yel, "New numerical results for the time-fractional phifour equation using a novel analytical approach," Symmetry, vol. 12, no. 478, pp. 1-16, 2020.

[5] Y. Zhang, C. Cattani, and X. J. Yang, "Local fractional homotopy perturbation method for solving," Non-Homogeneous Heat Conduction Equations in Fractal Domains, vol. 17, no. 10, pp. 6753-6764, 2015.

[6] W. Gao, P. Veeresha, D. G. Prakasha, H. M. Baskonus, and G. Yel, "New approach for the model describing the deathly disease in pregnant women using Mittag-Leffler function," Chaos, Solitons \& Fractals, vol. 134, Article ID 109696, 2020.

[7] WHO, Hepatitis B.WHO, Geneva, Switzerland, 2017, http:// www.who.int/news-room/fact-sheets/detail/hepatitis-b.

[8] K. Manna and S. P. Chakrabarty, "Global stability and a nonstandard finite difference scheme for a diffusion driven HBV model with capsids," Journal of Difference Equations and Applications, vol. 21, no. 10, pp. 918-933, 2015.

[9] K. Manna and S. P. Chakrabarty, "Chronic hepatitis B infection and HBV DNA-containing capsids: modeling and analysis," Communications in Nonlinear Science and $\mathrm{Nu}$ merical Simulation, vol. 22, no. 1-3, pp. 383-395, 2015.

[10] T. Guo, H. Liu, H. Liu, C. Xu, and F. Yan, "Global stability of a diffusive and delayed HBV infection model with HBV DNAcontaining capsids and general incidence rate," Discrete \& Continuous Dynamical Systems-B, vol. 23, no. 10, pp. 42234242, 2018.

[11] K. Hattaf and N. Yousfi, Global Properties of a Diffusive HBV Infection Model with Cell-To-Cell Transmission and Three Distributed Delays, Disease Prevention and Health Promotion in Developing Countries, Springer, Cham, Switzerland, 2020.

[12] M. Bachraou, K. Hattaf, and N. Yousfi, "A fractional order model for HBV infection with capsids and cure rate," in Trends in Biomathematics: Mathematical Modeling for Health,
Harvesting, and Population Dynamics, R. Mondaini, Ed., pp. 359-371, Springer, Cham, Switzerland, 2019.

[13] K. Manna and S. P. Chakrabarty, "Dynamics and analysis of a model for chronic hepatitis B infection," Journal of Interdisciplinary Mathematics, vol. 20, no. 2, pp. 339-355, 2017.

[14] X. Zhou and Q. Sun, "Stability analysis of a fractional-order HBV infection model," International Journal of Applied Mathematics and Mechanics, vol. 2, no. 2, pp. 1-6, 2014.

[15] S. M. Salman and A. M. Yousef, "On a fractional-order model for HBV infection with cure of infected cells," Journal of the Egyptian Mathematical Society, vol. 25, pp. 445-451, 2017.

[16] L. C. Cardoso, F. L. P. Dos Santos, and R. F. Camargo, "Analysis of fractional-order models for hepatitis B," Computational and Applied Mathematics, vol. 37, no. 4, p. 4570, 2018.

[17] K. Hattaf and N. Yousfi, "A class of delayed viral infection models with general incidence rate and adaptive immune response," International Journal of Dynamics and Control, vol. 4, no. 3, pp. 254-265, 2016.

[18] J. R. Beddington, "Mutual interference between parasites or predators and its effect on searching efficiency," The Journal of Animal Ecology, vol. 44, no. 1, pp. 331-340, 1975.

[19] D. L. DeAngelis, R. A. Goldstein, and R. V. O’Neill, “A model for tropic interaction," Ecology, vol. 56, no. 4, pp. 881-892, 1975.

[20] K. Hattaf, N. Yousfi, and A. Tridane, "Stability analysis of a virus dynamics model with general incidence rate and two delays," Applied Mathematics and Computation, vol. 221, pp. 514-521, 2013.

[21] A. A. Arafa, S. Z. Rida, and M. Khalil, "A fractional-order model of HIV infection: numerical solution and comparisons with data of patients," International Journal of Biomathematics, vol. 7, no. 4, pp. 1-11, 2014.

[22] C. V. De-Leon, "Volterra-type Lyapunov functions for fractional-order epidemic systems," Communications in Nonlinear Science and Numerical Simulation, vol. 24, pp. 75-85, 2015.

[23] J. Huo, H. Zhao, and L. Zhu, "The effect of vaccines on backward bifurcation in a fractional order HIV model," Nonlinear Analysis: Real World Applications, vol. 26, pp. 289-305, 2015.

[24] I. Petras, "Fractional derivatives, fractional integrals, fractional differential equations in matlab," in Engineering Education and Research Using MATLABInTech, London, UK, 2011. 